\title{
EFECTO NEUROTÓXICO DEL EXTRACTO ACUOSO DE BOLDO (Peumus boldus) EN UN MODELO ANIMAL
}

\author{
Jhon William Mejía-Dolores, 1,2,a, Daniel Enrique Mendoza-Quispe ${ }^{1, a}$, Edwin Luis Moreno-Rumay 1,3,a, \\ Carlos Alejandro Gonzales-Medina, ${ }^{1,3, b}$, Fany Remuzgo-Artezano ${ }^{1,3, b}$, Luis Alexander Morales-Ipanaqué1,a, \\ Roberto Carlos Monje-Nolasco 1,3,a
}

RESUMEN

Objetivos. Evaluar el efecto neurotóxico del extracto acuoso de boldo (Peumus boldus) en un modelo experimental. Materiales y métodos. Se diseñó un experimento, que incluyó 20 ratas macho Holtzman de $250 \pm 15 \mathrm{gr}$, distribuidas aleatoriamente en cuatro grupos: el control negativo recibió solución salina (SS) por vía oral (VO), el control positivo que recibió 6-hidroxidopamina por vía intracraneal (VIC) y SS por VO, el grupo experimental 1 recibió extracto acuoso de boldo (EAB) por VO y el grupo experimental 2 recibió 6-hidroxidopamina por VIC y EAB por VO, en todos los casos durante 21 días. Se realizó una evaluación neurológica, la cual tuvo tres componentes: a) clínico, evaluado con el test de rotarod, b) bioquímico, mediante la determinación de niveles séricos de ácido úrico, y c) histopatológico, por inmunohistoquímica para neuronas dopaminérgicas de sustancia negra. Se empleó la prueba de Kruskal Wallis y el test de Dunn para evaluar las diferencias entre los grupos. Resultados. Se encontró disminución significativa en el tiempo de latencia del test de rotarod entre los grupos control negativo y control positivo $(p<0,01)$, control negativo y experimental $1(p=0,09)$, control negativo y experimental $2(p<0,01)$, control positivo y experimental $1(p=0,04)$, y experimental 1 y $2(p=0,09)$. En la determinación de ácido úrico no hubo diferencia significativa intergrupal. En el conteo neuronal hubo depleción de neuronas dopaminérgicas totales, pero sin diferencia intergrupal. Conclusiones. Se evidencio un efecto neurotóxico del extracto acuoso de boldo en ratas macho de la cepa Holtzman a nivel clínico.

Palabras clave: Peumus; Neurotoxinas; Hidroxidopaminas; Trastornos parkinsonianos; Experimentación animal (fuente: DeCS BIREME)

\section{NEUROTOXIC EFFECT OF AQUEOUS EXTRACT OF BOLDO (Peumus boldus) IN AN ANIMAL MODEL}

\begin{abstract}
Objectives. To assess the neurotoxic effect of the aqueous extract of boldo (Peumus boldus) in an experimental model. Materials and methods. 20 male Holtzman rats of $250 \pm 15 \mathrm{~g}$ were randomized into four groups: the negative control received saline solution (SS) orally (PO), the positive control received 6-hydroxydopamine intracranially (IC) and SS by PO. Experimental group 1 received aqueous extract of boldo (AEB) by PO, and experimental group 2 received 6-hydroxydopamine intracranially and AEB by PO. The experiment lasted 21 days. A neurological assessment was performed which had three components: a) clinical, evaluated with the rotarod test, b) biochemical, by measuring serum levels of uric acid, and c) histopathology, by immunohistochemistry for substantia nigra dopaminergic neurons. The Kruskal Wallis test and the Dunn test were used to assess differences between groups. Results. A significant decrease was found in the latency time of the rotarod test between the negative and positive control group $(p<0.01)$, negative control and experimental $1(p=0.09)$, negative control and experimental $2(p<0.01)$, positive control and experimental $1(p=0.04)$, and experimental 1 and $2(p=0.09)$. There was no significant intergroup difference in the identification of uric acid. There was a depletion of the total dopaminergic neurons in the neuronal count, without intergroup difference. Conclusions. A neurotoxic effect of aqueous extract of boldo was recognized at a clinical level in Holtzman male rats.
\end{abstract}

Key words: Peumus; Neurotoxins; Hydroxydopamines; Parkinsonian disorders; Animal experimentation (Source: MeSH)

\footnotetext{
Facultad de Medicina, Universidad Nacional Mayor de San Marcos. Lima, Perú.

Sociedad Científica San Fernando, Universidad Nacional Mayor de San Marcos. Lima, Perú

Asociación para el Desarrollo de la Investigación en Ciencias de la Salud (ADIECS). Lima, Perú.

Estudiante de Medicina; ${ }^{\mathrm{b}}$ Médico cirujano

Recibido: 03-07-13 Aprobado: 16-10-13
}

Citar como: Mejía-Dolores JW, Mendoza-Quispe DE, Moreno-Rumay EL, Gonzales-Medina CA, Remuzgo-Artezano F, Morales-Ipanaqué LA, et al. Efecto neurotóxico del extracto acuoso de boldo (Peumus boldus) en un modelo animal. Rev Peru Med Exp Salud Publica. 2014;31(1):62-8. 


\section{INTRODUCCIÓN}

La enfermedad de Parkinson (EP) es un trastorno neurodegenerativo debido a una pérdida progresiva de neuronas dopaminérgicas del sistema nigroestrial, cuya manifestaciones clínicas principales son la bradicinesia, la rigidez y el tremor (1). Es la segunda enfermedad neurodegenerativa más frecuente en el mundo, y afecta a cerca del $3 \%$ de la población mayor de 65 años y a casi un $10 \%$ de los mayores de 80 años ${ }^{(2)}$. Además, se ha estimado que el número de personas con EP mayores de 50 años en los cinco países más poblados de Europa occidental y en los diez países más poblados del mundo, fue de 4,1 a 4,6 millones en el 2005 y que este valor se duplicaría para el año $2030{ }^{(3)}$. Sobre su patogenia, se conoce que, al menos, un $90 \%$ es idiopática (4); sin embargo, se ha descrito que el estrés oxidativo estaría fuertemente relacionado ${ }^{(5)}$. Esto ha dado lugar a la búsqueda de opciones terapéuticas con antioxidantes neuroprotectores que retrasen la muerte neuronal ${ }^{(6,7)}$.

El Peumus boldus (boldo) pertenece a la familia Monimiácea y es propia de las regiones andinas de Chile, Perú y Ecuador. Sus hojas son comúnmente empleadas en la medicina tradicional para problemas asociados al hígado. Presenta entre sus componentes la boldina, flavonoides y catequinas (8,9) las cuales han demostrado efectos benéficos, sobre todo, como antiinflamatorios y antioxidantes, por lo cual podrían influir en el estrés oxidativo presente en la EP ${ }^{(10)}$. Sin embargo, también presenta cantidades considerables de tetrahidroisoquinolonas (11), una neurotoxina que se encuentra en pesticidas y que ha sido firmemente asociada al desarrollo de EP ${ }^{(12,13)}$.

Según la Organización Mundial de la Salud, más de $80 \%$ de la población mundial usa preparaciones botánicas como medicina tradicional, dentro de ellas el Peumus boldus. Evidentemente, este uso se hace sin conocer los efectos negativos en el ámbito neurológico que esta produciría ${ }^{(14-16)}$. El objetivo del estudio fue evaluar el efecto neurotóxico que produce el Peumus boldus en ratas.

\section{MATERIALES Y MÉTODOS}

El estudio experimental se llevó a cabo en los ambientes de la Facultad de Medicina y de Farmacia y Bioquímica de la Universidad Nacional Mayor de San Marcos (UNMSM) y en el Laboratorio de Neurociencias y Comportamiento de la Universidad Peruana Cayetano Heredia (UPCH) durante los meses de octubre a diciembre de 2011.

\section{PREPARACIÓN DEL EXTRACTO ACUOSO DE BOLDO}

Las plantas de Peumus boldus $L$. fueron adquiridas en un mercado mayorista. La certificación taxonómica de ellas fue realizada por el Museo de Historia Natural de la UNMSM (Constancia 154 USM-2011). Para la preparación del extracto acuoso de boldo (EAB) se secaron las hojas de Peumus boldus $L$. en una cámara de secado durante cinco días, para luego pulverizarlas en molino eléctrico. El material resultante fue diluido en agua a $100^{\circ} \mathrm{C}$ y macerado por $6 \mathrm{~h}$. Para eliminar el posible detritus existente, la disolución fue filtrada, luego de lo cual fue colocada en un recipiente en una estufa a $37^{\circ} \mathrm{C}$, para retirar el solvente. La película fina obtenida fue separada del recipiente, luego diluida en suero salino y almacenada en un frasco ámbar. La determinación cualitativa de alcaloides se realizó con los reactivos de Dragendorf; la de alcaloides, con la de Mayer, y la de flavonoides, con la de Shinoda.

\section{MODELO ANIMAL}

Se emplearon veinte ratas macho Holztman de 3 meses de edad, con un peso de $250 \pm 15 \mathrm{~g}$, procedentes del bioterio de la Universidad Nacional Agraria La Molina, las cuales fueron mantenidas a $22 \pm 1{ }^{\circ} \mathrm{C}$, en un ciclo de $12 \mathrm{~h}$ luz/oscuridad, y recibieron agua y alimento ad libitum. Ellas fueron aclimatadas por 7 días, sin ser sometidas a ningún tipo de intervención. Luego de la aclimatación, las ratas fueron distribuidas aleatoriamente en cuatro grupos y recibieron el siguiente tratamiento: el primer grupo, el control blanco, recibió solución salina (SS) por vía oral (VO) durante 21 días; el segundo grupo, control positivo, recibió 6-hidroxidopamina (6-OHDA) a razón de 2 ug/uL por vía intracraneal $(\mathrm{VIC})$ por cirugía estereotáxica y SS por VO por 21 días; el tercer grupo, o experimental 1, recibió $\mathrm{EAB}$ a razón de de $200 \mathrm{mg} / \mathrm{kg} / \mathrm{día}$ por VO por 21 días; y el cuarto grupo, experimental 2, recibió 6-OHDA a razón de 2 ug/uL por VIC por cirugía estereotáxica y EAB a razón de $200 \mathrm{mg} / \mathrm{kg} / \mathrm{día}$ por VO por 21 días. Para la administración del suero salino y del EAB se empleó una cánula orogástrica, esta se inició dos días después de realizada la cirugía estereotáxica, por 21 días.

Cirugía estereotáxica para inyección intracraneal de 6-OHDA. La ratas fueron anestesiadas con inyección intraperitoneal de clorhidrato de ketamina $5 \mathrm{mg} / \mathrm{kg}$, clorhidrato de xilazina $1 \mathrm{mg} / \mathrm{kg}$ y sulfato de atropina $0,05 \mathrm{mg} / \mathrm{kg}$ (Ketaxy ${ }^{\circledR} 0,25 \mathrm{ml} / \mathrm{kg}$ ); y colocadas en el estereotáxico. Previa administración de atropina vía tópica (para evitar sangrado) se rasuró la zona frontal de la cabeza de las ratas y se hizo una incisión anteroposterior hasta exponer el cráneo. Para la determinación del área de punción se ubicaron las coordenadas de la pars compacta derecha de la sustancia negra conforme al atlas de Paxinos y 
Watson: bregma anteroposterior $-5,0 \mathrm{~mm}$; lateral 2,1 mm; y 7,7 mm de profundidad desde la duramadre. Se taladró el cráneo hasta la duramadre y con una jeringa Hammilton se inyectó unilateralmente (lado derecho) $4 \mu \mathrm{g}$ de 6-OHDA (Sigma-Aldrich) disuelta en $2 \mu \mathrm{l}$ de ácido ascórbico $0,2 \mathrm{mg} / \mathrm{mL}$, a una frecuencia de $0,5 \mu \mathrm{L} /$ min. Finalmente, se aplicó metil metacrilato vía tópica para cubrir el cráneo y, una vez secado, se suturó $(16,17)$.

\section{EVALUACIÓN NEUROLÓGICA}

Para evaluar con la mayor amplitud un posible daño dopaminérgico, la evaluación neurológica incluyó tres componentes: a)componente clínico, el cual fue evaluado con el test de rotarod que correlaciona el déficit motor con el tamaño de la lesión ${ }^{(19)}$; b) el componente bioquímico, el cual fue evaluado a través de la determinación de ácido úrico sérico, el cual es un marcador oxidativo que, se ha visto, se encuentra disminuido en la EP (20,21), y c) el componente histopatológico, que fue evaluado con inmunohistoquímica para neuronas dopaminérgicas positivas a tirosin hidroxilasa $(\mathrm{TH}+)$ y permite la correcta evaluación histopatológica de la sustancia negra. Las consideraciones tomadas durante estas evaluaciones se detallan a continuación.

Test de rotarod. Las mediciones se realizaron los días 7 y 19, luego del inicio de la administración del EAB. El dispositivo cuenta con un eje de rotación de $7,3 \mathrm{~cm}$ de diámetro y un carril de rodillos que gira a 20 revoluciones por minuto. Se contabilizó el tiempo desde que es colocada la rata en el rodillo hasta que cae de este, los datos se presentan como de tiempo de latencia de caída (segundos).

Medición sérica de ácido úrico. Las mediciones se realizaron los días 11 y 19, luego del inicio de la administración de EAB; se aplicó a la rata anestesia inhalatoria con éter y se obtuvo muestras de sangre, por método de punción cardiaca. Esta muestra se centrifugó y se trabajó con el suero. A $0,025 \mathrm{~mL}$ del suero se agregó $1 \mathrm{~mL}$ de reactivo líquido para determinación enzimática de ácido úrico en suero (Baltex); se incubó por 5 min a $37{ }^{\circ} \mathrm{C}$ y se llevó a lectura de absorbancias en espectrofotómetro. Con ello se hizo el cálculo de concentración de ácido úrico sérico. Los datos se presentaron en miligramos por decilitro (mg/dL).

Inmunohistoquímica. Al finalizar la administración de los solutos. Una rata de cada grupo fue anestesiada con pentobarbital sódico, el cual fue administrado por vía intraperitoneal a razón de 0,6 $\mathrm{mL} / \mathrm{kg}$, y perfundida transcardialmente con $150 \mathrm{~mL}$ de solución salina, seguido por $240 \mathrm{~mL}$ de paraformaldehído al $4 \%$ durante $30 \mathrm{~min}$. Tras lo cual, los cerebros fueron removidos, fijados en formol al $10 \%$ por 2 semanas y posfijados por inmersión en formol al 10\%. Las secciones de parafina de 5 um de espesor, obtenidas con micrótomo, fueron lavadas tres veces por 5 min en tampón fosfato salino $(10 \mathrm{mM}, \mathrm{pH}=7,4)$ y se trataron con $\mathrm{H}_{2} \mathrm{O}_{2}$ por 5 min. Siguió la preincubación de 30 min con suero normal de cabra al $10 \%$. Luego, las secciones fueron incubadas con anticuerpo anti-TH (1:200), incluyendo Tritón X-100 al $0,3 \%$ en la noche a $4{ }^{\circ} \mathrm{C}$. Después de un enjuague en tampón fosfato salino $(10 \mathrm{mM}, \mathrm{pH}=7,4)$ por $15 \mathrm{~min}$, las secciones se incubaron con anticuerpo secundario avidine-biotinperoxidasa por $30 \mathrm{~min}$ a temperatura ambiente. Finalmente, la inmunorreactividad fue detectada mediante el kit Vectastain Elite ABC (PROLAB, Chile) y diaminobencidina. Las microfotografías fueron tomadas con lente objetivo de 10x, en microscopio óptico. Se contabilizó el número de neuronas dopaminérgicas $\mathrm{TH}+$ con la versión libre del software JMicrovision ${ }^{\circledR}$ versión 1.2.7.

\section{ANÁLISIS ESTADÍSTICO}

La descripción de variables se realizó con medianas y rango intercuartílico. Para la comparación estadística entre grupos se usó la prueba de Kruskal Wallis, y la prueba pos hoc test de Dunn para saber entre qué grupos hay diferencia. Se consideró un valor $p<0,05$ como significativo. Se usó el software estadístico SPSS Versión 20.0 (Trial).

\section{ASPECTOS ÉTICOS}

Los animales fueron manejados de acuerdo con la Guide for the Care and Use of Laboratory Animals ${ }^{(22)}$. Se utilizó Ketaxyl ${ }^{\circledR}$, una combinación de ketamina, xilazina y atropina, que brinda una adecuada inducción de la anestesia, anestésico, tranquilizante y anticolinérgico en procedimientos quirúrgicos de animales de experimentación ${ }^{(23,24)}$.

\section{RESULTADOS}

Con relación a la evaluación clínica, los resultados expresados, como el tiempo de latencia (segundos) en el test de rotarod, se presentan en la Figura 1, en donde se observa un tiempo de latencia disminuido luego de la administración del EAB. Durante la segunda medición del test de rotarod, se encontró diferencias al comparar las medianas del tiempo de latencia $(p=0,04$, Kruskal Wallis). Se encontró diferencias entre el control negativo y el control positivo ( $p<0,01$, test de Dunn), entre el control negativo y el grupo experimental $1(p=0,09$, test de Dunn), entre el control negativo y el grupo experimental 2 ( $p<0,01$, test de Dunn), y entre el grupo experimental 1 y el grupo experimental 2 ( $p=0,09$, test de Dunn). 


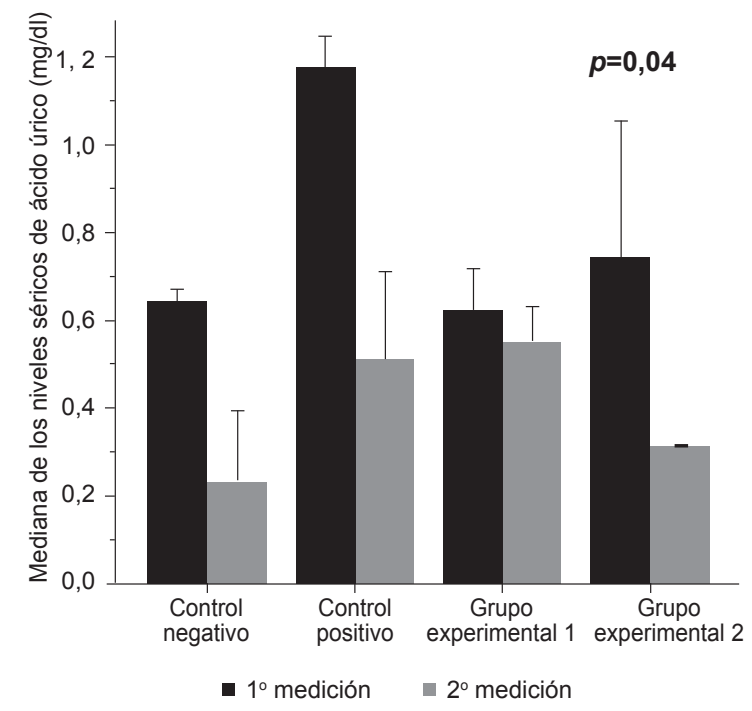

Figura 1. Evaluación clínica (test de rotarod) del daño neurotóxico mediado por Peumus boldus L. (boldo) en un modelo experimental de parkinson inducido por 6-hidroxidopamina

Nota. Se muestran las mediciones llevadas a cabo a los 7 y 19 días del inicio de la administración de las sustancias.

Control negativo: solución salina vía oral (VO); control positivo: 6-hidroxidopamina intracraneal (IC); grupo experimental 1: extracto acuoso de boldo (EAB) VO; grupo experimental 2: EAB VO + 6-hidroxidopamina IC

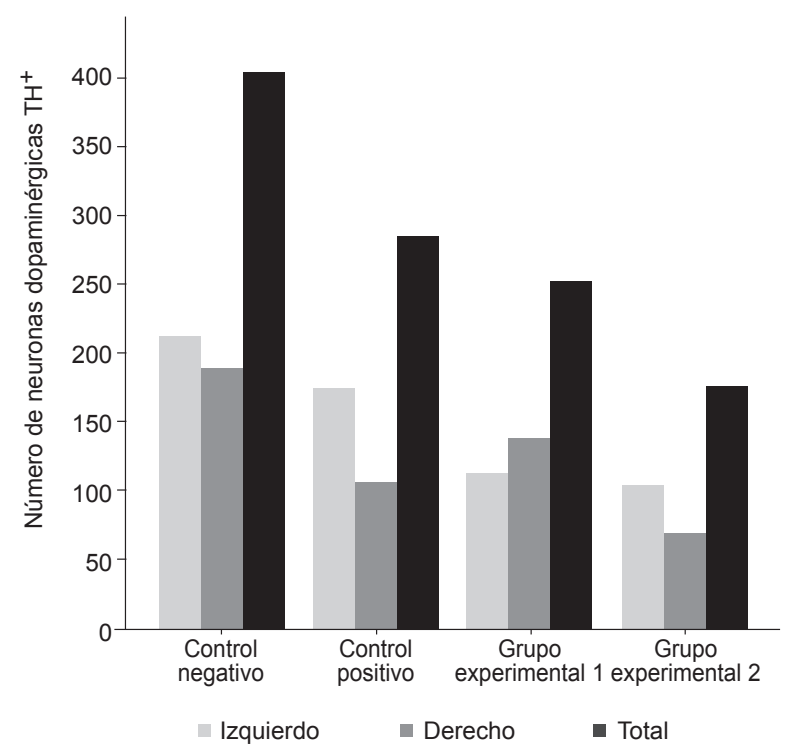

Figura 3. Evaluación inmunohistoquímica (cuantificación de células $\mathrm{TH}+$ ) del daño neurotóxico mediado por Peumus boldus $L$. (boldo) en un modelo experimental de parkinson inducido por 6-hidroxidopamina

Nota. La neurotoxicidad es evaluado por el número de neuronas dopaminérgicas sobrevivientes a la administración de sustancias cuantificadas con el software JMicrovisión ${ }^{\circledR}$.

Control negativo: solución salina vía oral (VO); control positivo: 6-hidroxidopamina intracraneal (IC); grupo experimental 1: extracto acuoso de boldo (EAB) VO; grupo experimental 2: EAB VO + 6-hidroxidopamina IC. TH+: anti tirosina hidroxilasa positivo.

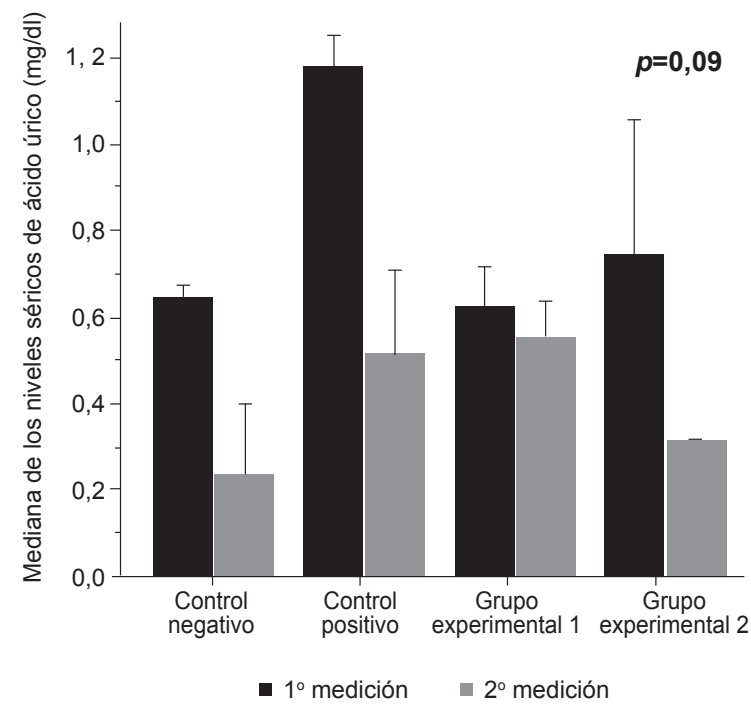

Figura 2. Evaluación bioquímica (determinación de niveles de ácido úrico) del daño neurotóxico mediado por Peumus boldus $L$. (boldo) en un modelo experimental de parkinson inducido por 6-hidroxidopamina

Nota. Se muestran las mediciones llevadas a cabo a los 11 y 19 días de inicio de la administración de las sustancias.

Control negativo: solución salina vía oral (VO); control positivo: 6-hidroxidopamina intracraneal (IC); grupo experimental 1: extracto acuoso de boldo (EAB) VO; grupo experimental 2: EAB VO + 6-hidroxidopamina IC.

No se encontró diferencias entre las medianas del control positivo y del grupo experimental $2(p=0,37$, test de Dunn). Respecto a la evaluación bioquímica, en la Figura 2 se presentan los resultados de ambas mediciones, no se encontró diferencia intergrupal ( $p=0,09$, Kruskal Wallis).

En la evaluación histopatológica, el conteo neuronal basado en la reactividad positiva a la tirosina hidroxilasa mostró una depleción de neuronas dopaminérgicas totales (Figura 3) en la pars compacta de la sustancia negra, en el control positivo, de $29,6 \%$, y del $37,9 \%$ en el grupo experimental 1, en comparación con el control negativo. En tanto, se observó un número neuronal $30 \%$ menor en el grupo experimental 2 comparado con el grupo experimental 1 (Figura 4).

\section{DISCUSIÓN}

El test de rotarod, que es ampliamente utilizado para evaluar el déficit motor en modelos de enfermedades neurodegenerativas en roedores, correlaciona los efectos de la coordinación motora con la disfunción de la dopamina y el tamaño de la lesión cerebral (18). Por ello, las ratas que reporten menores tiempos de latencia indicarán mayor pérdida neuronal. Como se reporta en estudios anteriores ${ }^{(18,22)}$, observamos que las ratas que 

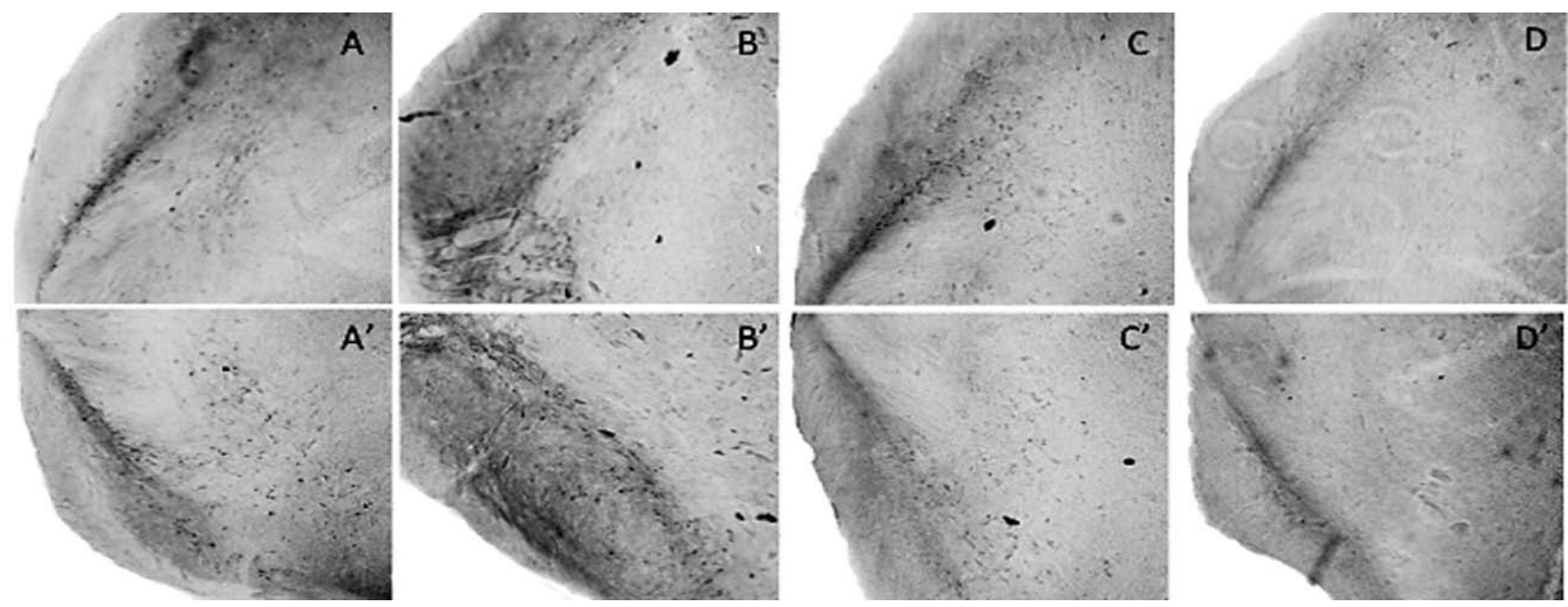

Figura 4. Fotomicrografías de cerebro (sustancia nigra pars compacta) de rata con daño neurotóxico mediado por Peumus boldus L. (boldo) en un modelo experimental de parkinson inducido por 6-hidroxidopamina (tinción inmunohistoquímica con anticuerpo policlonal de conejo anti tirosina hidroxilasa $(\mathrm{TH}+)$ de los grupos, 10X)

Control negativo: solución salina (A, $\left.A^{\prime}\right)$; control positivo: 6-hidroxidopamina intracraneal (B,B'); grupo experimental 1: extracto acuoso de boldo (EAB) (C,C'); grupo experimental 2: EAB + 6-hidroxidopamina (D,D'). Nótese la pérdida de densidad de células TH+ en el control positivo (B'), grupo experimental 1 (C') y 2 ( $\left.D^{\prime}\right)$.

recibieron la neurotoxina (6-OHDA) tuvieron un menor tiempo de latencia que el grupo control negativo, lo cual sería consecuencia del daño dopaminérgico producido por esta neurotoxina. Los tiempos de latencia obtenidos, luego de la administración de EAB, aunque no son significativos, muestran una existencia de daño en la coordinación motora en el grupo que solo recibió el EAB, comparado con el control negativo. Similar relación se encuentra entre los grupos que recibieron el EAB y el 6-OHDA, lo que indicaría que el daño producido por ambos es similar. Es probable que esta diferencia se vea influida por el tamaño de la muestra. A pesar de ello, la tendencia observada en la Figura 2, sugiere un probable efecto neurotóxico del boldo, el cual consideramos sería más evidente si la exposición al boldo hubiese sido más prolongada.

Estudios anteriores indican la presencia en las hojas del boldo, de tetrahidroisoquinolonas, sustancias fuertemente asociadas con neurotoxicidad (11,13). Estas sustancias, según DeCuypere ${ }^{(25)}$, estarían involucradas en el proceso de muerte celular monoaminérgica estudiado en modelos de EP inducido. En otro estudio, Scholz (12) indica que las tetrahidroisoquinolonas endógenas logran ligarse al hierro del centro catalítico de la tirosina hidroxilasa, enzima clave en la síntesis de dopamina a nivel neuronal, de una forma mimética a las catecolaminas en el proceso de regulación dopaminérgica por feedback, logrando así la inhibición de esta enzima y, por ende, la disminución de producción de dopamina neuronal.

Por otro lado, el ácido úrico $(\mathrm{AU})$ es un conocido antioxidante natural presente en la sangre y el tejido cerebral, sus acciones como antioxidante pueden regular el daño cerebral inducido por el estrés oxidativo, y esto puede explicar por qué se encuentra bajos niveles de esta sustancia en el suero de pacientes con EP ${ }^{(19,26)}$. Zhu et al. (27) demostraron que el $\mathrm{AU}$, como pretratamiento, era capaz de proteger las células PC12 contra la lesión inducida por 6-OHDA in vitro. Asimismo, Gong L. et al. ${ }^{(28)}$ también sostienen que el aumento de los niveles del antioxidante urato puede proteger contra la EP, y aseguran que los ratones con una mutación genética, que condicionaba el incremento en los niveles de ácido úrico, fueron protegidos contra el tipo de neurodegeneración que subyace a la EP, mientras que este tipo de daño fue más notorio en animales con ácido úrico anormalmente bajo. Toda esta evidencia coloca actualmente al ácido úrico como un importante biomarcador del estrés oxidativo relacionado con la patogenia de la EP ${ }^{(29)}$. Si bien en nuestro estudio no se encontraron diferencias en los niveles de ácido úrico entre los grupos en ambas mediciones, se puede observar, en la primera medición, que el grupo que recibió solo el EAB presentó menores niveles de $A U$ que el grupo control negativo, lo cual se atribuiría a un mayor estrés oxidativo por la administración del extracto acuoso de boldo. También apoya a un posible efecto neurotóxico del boldo el hecho que se obtuvieran menores niveles de $\mathrm{AU}$ en ambos grupos experimentales, es decir, en aquellos animales que recibieron el EAB ya sea solo, o de manera conjunto con el 6-OHDA- al comparar la segunda medición con respecto a la primera.

Con relación a la evaluación inmunohistoquímica, la depleción de células $\mathrm{TH}+$ en el control positivo con respecto al negativo, mostró una muerte neuronal 
atribuible al efecto neurotóxico de la 6-OHDA, con una menor intensidad vista en el estudio de Thornton (30), esta misma situación ocurre al comparar con el hemisferio contralateral no lesionado de dicho grupo, lo que puede ser atribuible a la menor cantidad de neurotóxico administrado. La pérdida del $37,9 \%$ de neuronas dopaminérgicas observado en las microfotografías del grupo que recibió el EAB, con respecto al control negativo, sugiere también un efecto neurotóxico del extracto. Más aun, la mayor pérdida de neuronas dopaminérgicas observada en el grupo que recibió tanto EAB como la 6-OHDA, comparada con los grupos que recibieron estas sustancias de manera independiente, sugiere que estas sustancias podrían presentar sinergismo para generar daño neurotóxico, por lo que tampoco se descarta la posibilidad de que estas presenten mecanismos de acción similares.

Algunas limitaciones deben ser reconocidas. En primer lugar, el no haber realizado una medición basal del AU previa a la administración del EAB o del 6-OHDA, de haberlo realizado se podría haber analizado la caída del ácido úrico en el control negativo en la segunda medición. Sin embargo, esta caída refleja la baja especificidad de esta prueba, y es que los nivel de AU, al ser un antioxidante, pueden alterarse por muchas otras situaciones oxidantes diferentes a la administración de 6-OHDA o de boldo. Otra limitación importante es la cantidad de ratas a la que se realizó la técnica de inmunohistoquímica, ello se debió básicamente a motivos económicos y administrativos, su existencia no permite inferir conclusiones más profundas en este aspecto por ello, deberá ser evaluado en posteriores estudios.
En conclusión, en condiciones experimentales se evidencia un efecto neurotóxico del extracto acuoso de boldo en ratas macho de la cepa Holtzman, el cual se encuentra presente únicamente en la evaluación clínica. Se requiere futuros estudios que evalúen el daño neurotóxico de una exposición más prolongada al Peumus boldus.

Agradecimientos: al PhD. Luis Ángel Aguilar Mendoza, coordinador del Laboratorio de Neurociencias y Comportamiento de la UPCH, por su asesoría y revisión del estudio; al Mg. Roy Andrade Espinoza, del Laboratorio de Neurociencias y Comportamiento de la UPCH por la realización de la técnica de inmunohistoquímica; al Dr. Jorge Arroyo Acevedo, docente de la sección de Farmacología de la UNMSM por la asesoría en la preparación del extracto acuoso de boldo; al Q.F. Rubén Valdivieso, del laboratorio del Instituto de Bioquímica y Nutrición de la UNMSM, por la colaboración en la medición del ácido úrico; al Lic. Walter, por la toma fotográfica de alta resolución con el uso del microscopio del Laboratorio de Inmunología del Servicio de Patología Clínica del Hospital Nacional Docente Madre-Niño San Bartolomé; al Sr. Renzo Vásquez por capacitarnos en la cirugía estereotáxica y la técnica de fijación por perfusión.

Contribuciones de autoría: JMD, DMQ, LMR, RMN, LMI, CGM y FRA contribuyeron con la concepción y el diseño del artículo. JMD, DMQ, LMQ, RMN y LMI participaron en la recolección y obtención de resultados, y en la obtención de financiamiento. JMD, DMQ, LMI, CGM, FRA participaron con el análisis e interpretación de datos, redacción del artículo y revisión crítica del artículo. JMD, DMQ y LMI aportaron el material de estudio. CGM y FRA brindaron asesoría estadística, técnica y administrativa. Todos los autores aprueban la versión final a publicar

Fuente de financiamiento: autofinanciado

Conflicto de Interés: los autores declaran no tener conflictos de interés.

\section{REFERENCIAS BIBLIOGRÁFICAS}

1. Decressac M, Pain S, Chabeauti PY, Frangeul L, Thiriet N, Herzog H, et al. Neuroprotection by neuropeptide $\mathrm{Y}$ in cell and animal models of Parkinson's disease. Neurobiol Aging. 2011;33(9): 2125-37.

2. Riedel O, Klotsche J, Spottke A, Deuschl G, Förstl H, Henn F, et al. Cognitive impairment in 873 patients with idiopathic Parkinson's disease. Results from the German Study on Epidemiology of Parkinson's Disease with Dementia (GEPAD). J Neurol. 2008;255(2):255-64.

3. Dorsey ER, Constantinescu R, Thompson JP, Biglan KM, Holloway RG, Kieburtz K, et al. Projected number of people with Parkinson disease in the most populous nations, 2005 through 2030. Neurology. 2007;68(5):384-6.

4. Weintraub D, Comella CL, Horn S. Parkinson's disease-Part 1: Pathophysiology, symptoms, burden, diagnosis, and assessment. Am J Manag Care. $2008 ; 14(2): 40-8$.

5. Martin HL, Teismann P. Glutathione-a review on its role and significance in Parkinson's disease. FASEB J. 2009;23(10):3263-72.

6. Uttara B, Singh AV, Zamboni P, Mahajan RT. Oxidative stress and neurodegenerative diseases: a review of upstream and downstream antioxidant therapeutic options. Curr Neuropharmacol. 2009;7(1):65-74.

7. Haleagrahara N, Siew CJ, Mitra NK, Kumari M. Neuroprotective effect of bioflavonoid quercetin in 6-hydroxydopamine-induced oxidative stress biomarkers in the rat striatum. Neurosci Lett. 2011;500(2):139-43. doi: 10.1016/j. neulet.2011.06.021.

8. Ochoa C, Granda C, Chapoñan M, Borja R, Borjas P, Ortiz J, Ugaz G, Puerta E, Pucutay M. Efecto Protector de Peumus boldus en ratas con toxicidad hepática inducida por Paracetamol. CIMEL. 2008;13(1):20-5. 
9. Yu B, Cook C, Santanam N. The aporphine alkaloid boldine induces adiponectin expression and regulation in 3T3-L1 cells. J Med Food. 2009;12(5):1074-83.

10. Brian P, Carrasco C, Speisky H. Boldine and its antioxidant or healthpromoting properties. Chem Biol Interact. 2006;159(1):1-17.

11. Chand P, Litvan I. Neurobiology of progressive supranuclear palsy. Neurobiol Dis. 2007;105-10.

12. Scholz J, Toska K, Luborzewski A, Maass A, Schunemann V, Haavik J, et al. Endogenous tetrahydroisoquinolines associated with Parkinson's disease mimic the feedback inhibition of tyrosine hidroxylase by catecholamines. FEBS J. 2008;275(9):2109-21.

13. Silva G, Hepp R, Tapia M, Casals P, Bustos G, Osses F. Evaluación de boldo (peumus boldus Molina) y cal para el control de Sitophilus zeamais Motschulsky. Agrociencia 2006;40(2):219-28.

14. Mukherjee P K, Wahile A. Integrated approaches towards drug development from Ayurveda and other Indian system of medicines. J Ethnopharmacol. 2006;103(1):25-35.

15. Avello M, Rivas X, Pastene E. Evaluación de plantas medicinales de uso común en la ciudad de Concepción (Chile), a través de ensayos de calidad. Boletín Latinoamericano y del Caribe de Plantas Medicinales y Aromática. 2011;10(4):379-88.

16. Avoy F, Sotelo C, Herbet A, Agid Y. Specificity of dopaminergic neuronal degeneration induced by intracerebral injection of 6-hydroxydopamine in the nigrostriatal dopamine system. Brain Res. 1976;102(2):201-15.

17. Paxinos G, Watson C. The Rat Brain in Stereotaxic Coordinates: Second edition. San Diego, CA: Academic Press; 1987.
18. Monville C, Torres E, Dunne S. Comparison of incremental and accelerating protocols of the rotarod test for the assessment of motor deficits in the 6-OHDA model. J Neurosci Methods. 2006;158(2):21923.

19. Annanmaki T, Muuronen A, Murros K. Low plasma uric acid level in Parkinson. Mov Disord. 2007;22(8):1133-7.

20. Licea T, Badía Ll, Borges L. Conservación de soluciones de referencia de ácido úrico. Rev Cubana Farm. 1997;31(2):102-6.

21. The National Research Council of The National Academy of Sciencies. Guide for the Care and Use of Laboratory Animals: Eight Edition. Washington, DC: The National Academies Press; 2010.

22. Schwarting R, Huston J. The unilateral 6-OHDA injection lesion model in behavior brain research. Analysis of functional deficit, recovery and treatments. Prog Neurobiol. 1996;50(2-3):275-331.

23. The University of Sidney, Laboratory Animal Services. Standard operating procedures: Anaesthesia and pain management - rodents. 8th Edition. Darlington New South Wales: The University of Sidney; 2012

24. Moore J, Haouzi P, Van de Louw A, Bell HJ. Hypocapnia-dependent facilitation of augmented breaths: observations in awake vs. anesthetized rats. Respir Physiol Neurobiol. 2012;180(1):105-11.

25. DeCuypere M, Lu Y, Miller DD, LeDoux MS. Regional distribution of tetrahydroisoquinoline derivatives in rodent, human, and Parkinson's disease brain. J Neurochem. 2008;107(5):1398-413.

26. Andreadou E, Nikolaou C, Gournaras F, Rentzos M, Boufidou F, Tsoutsou $A$, et al. Serum uric acid levels in patients with Parkinson's disease: their relationship to treatment and disease duration. Clin Neurol Neurosurg. 2009;111(9):724-8.

27. Zhu TG, Wang XX, Luo WF, Zhang QL, Huang TT, Xu XS, et al. Protective effects of urate against 6-OHDAinduced cell injury in $\mathrm{PC} 12$ cells through antioxidant action. Neurosci Lett. 2011;506(2):175-9. doi: 10.1016/j.neulet.2011.10.075.

28. Gong L, Zhang QL, Zhang N, Hua WY, Huang YX, Di PW, Huang $\mathrm{T}$, et al. Neuroprotection by urate on 6-OHDA-lesioned rat model of Parkinson's disease: linking to Akt/GSK3 $\beta$ signaling pathway. J Neurochem. 2012;123(5):876-85.

29. Cipriani S, Chen X, Schwarzschild M. Urate: a novel biomarker of Parkinson's disease risk, diagnosis and prognosis. Biomark Med. 2010;4(5):701-12.

30. Thornton E, Vinck R. Treatment with a Substance P Receptor Antagonist Is Neuroprotective in the Intrastriatal 6-Hydroxydopamine Model of Early Parkinsons's Disease. PLos One. 2012;7(4):e34138. Doi: 10.1371/ journal.pone. 0034138

Correspondencia: Jhon Mejía Dolores Dirección: Jr. Zorritos 1399, block 4, departamento 402, Lima 1, Perú. Teléfono: 942-116275

Correoelectrónico:jmejiad3@gmail.com 